Fátima Barbosa, Cláudia Cunha, Gina Voss

and Alice Delerue Matos

\title{
25 The impact of living alone on physical and mental health: Does loneliness matter?
}

\footnotetext{
- The number of people living alone is increasing among those aged 50 and older in Europe, but the proportions vary across countries

- Loneliness is a key factor in explaining the impact of living alone on health

- When loneliness is taken into account, solo living is not necessarily detrimental to the health
}

\subsection{Living alone, health and loneliness after $\mathbf{5 0}$}

Living alone is an increasingly common phenomenon in ageing populations. The death of a spouse and the tendency of older people and their adult children to maintain independent lifestyles are noted as the main causes for this gradual increase in solo living in recent decades (Victor et al., 2000). However, the impact of living alone on the physical and mental health of older people is still unclear.

Research on this subject shows that older people who live alone have a high risk of experiencing loneliness (Park et al., 2017). As noted by ShiovitzEzra (2015), loneliness is defined as a feeling of distress that stems from the perception that the quantity and/or the quality of one's social ties is/are insufficient. A review of several studies concluded that loneliness is associated with poor physical and mental health (Courtin and Knapp, 2017; Shiovitz-Ezra, 2015). Given the increase in solo living among older Europeans, it is important to study the impact of living alone on their physical and mental health, taking into account the role of loneliness in this relationship over time.

\subsection{Study methods}

Our study sample included Europeans aged 50 years and older residing in the 16 European countries that participated in SHARE Waves 4, 5 and 6

Ә Open Access. (C 2019 Fátima Barbosa, et al., published by De Gruyter. (cc)BY-NC-ND This work is licensed under a Creative Commons Attribution-NonCommercial-NoDerivatives 4.0 International License. 
(Austria, Germany, Sweden, Netherlands, Spain, Italy, France, Denmark, Switzerland, Belgium, Czech Republic, Poland, Luxembourg, Portugal, Slovenia and Estonia) and in Israel. Because Luxembourg and Israel did not participate in Wave 4, the baseline measurement for these countries is Wave 5.

Our independent variable of primary interest - living alone - was categorized as 0 if the respondent's household size was two or more and 1 if the household size was one. The dependent variables were physical and mental health. For physical health, we created a latent continuous measure based on the procedures employed by Ploubidis and Grundy (2011). This measure combines an objective health indicator (maximum grip strength) and six selfreports. The self-reports were self-perceived health (a 5-point ordinal scale that ranged from poor (1) to excellent (5); the presence of long-term illness, coded 0 if yes and 1 if no; limited activities due to poor health: coded 1 if severely limited, 2 if limited but not severely and 3 if not limited; and three health conditions: (a) heart attack, (b) stroke and (c) chronic lung disease - all coded 0 if yes and 1 if no. According to Ploubidis and Grundy (2011), this indicator is less subject to measurement error and has greater reproducibility and reliability compared with individual health indicators used separately. To construct this variable, we used the statistical programme MPLUS, version 7, WLSMV estimator. The combined SHARE-based health indicator revealed a good model fit in all waves (RMSEA $=0.03$ in Wave 4, 0.03 in Wave 5 and 0.03 in Wave 6; CFI $=0.99$ in Wave 4, 0.99 in Wave 5 and 0.98 in Wave 6; and TLI $=0.98$ in Wave 4, 0.98 in Wave 5 and 0.97 in Wave 6).

Mental health was assessed using the EURO-D scale that counts up to 12 depressive symptoms, such as irritability, tearfulness and loss of enjoyment during the last month. The range of this scale is $0-12$. We employed a dummy dichotomous variable in which a EURO-D score of greater than three indicated clinical depression (1) and a score equal to or less than three indicated no depression (0).

This study was carried out in several stages. First, at baseline, we examined the percentages of individuals aged 50+ who lived alone in each country. Second, to assess the differences in physical and mental health at baseline between respondents living alone and those not living alone, we applied statistical tests for a two-group comparison (t-test $(\mathrm{t})$; chi-square tests $\left.\left(\mathrm{X}^{2}\right)\right)$. Effect size measures (Hedges' g/Phi) were used to complement these analyses. Lastly, two longitudinal linear mixed models were tested to analyse the effect of loneliness vis-à-vis the impact of living alone on the physical and mental health of the $50+$ population.

The first longitudinal model was adjusted for the following variables: age at the time of the interview, gender, retirement status (retired was coded as 1 
and all other categories as 0 (employed or self-employed, unemployed, permanently sick or disabled, homemaker and other) and financial distress (with a range from (1), 'makes ends meet easily', to (4) 'makes ends meet with great difficulty'). Educational level was divided into three categories based on the International Standard Classification of Education (ISCED): primary schooling or less (ISCED-97 score $=0-2$ ), secondary education (ISCED-97 score $=3$ ) and post-secondary education (ISCED-97 score $=4-6$ ). Also included in the model were the number of visits to the doctor in the previous month; physical inactivity (coded 1 if the respondent never practised moderate or vigorous physical activity and 0 if otherwise) and the number of activities done in the last year. Combining data from Waves 4, 5 and 6, for this first model, we have 175,717 observations for 99,021 individuals.

The second model was adjusted for all previously mentioned variables plus loneliness, assessed on the revised UCLA scale. The scale measures three general feelings of loneliness or feeling left out, lack of companionship and isolation. Each item was scored on a 3-point Likert scale (1 = Hardly ever or never; 2 = Some of the time; 3 = Often). Hence, the total scale score ranged from 3 to 9 . For the second model, we have 163,412 observations for 98,632 individuals.

\subsection{Results of the study}

Figure 25.1 shows a variation in living alone across the countries at baseline. Sweden (37\%), Estonia (32.8\%), Austria (32.2\%) and the Netherlands (30.7\%) were the countries with the highest percentage of people aged 50+ and living alone. Portugal (11.1\%), Poland (17.6\%), Spain (19.3\%) and Israel (19.4\%) had the lowest percentage.

Regarding physical health outcome, in all countries, people living alone had poorer health compared with those living with others (t-test, sig. $<0.001$ ). However, when considering effect size, which measures the magnitude of the differences found, only in Austria (0.27), Sweden (0.28), Italy (0.21), Denmark (0.35) and Switzerland (0.26) were the differences in physical health between these two types of living arrangements significant (for the t-test, the effect size used is Hedges' g: small effect $\geq 0.20$ but less than 0.50 ). In terms of mental health, in all countries, people living alone showed more severe depression (chi-square, sig < 0.10). Nevertheless, the effect size was significant only in Spain (0.11), Italy (0.12), Israel (0.11) and the Czech Republic (0.11). (For chi-square, the effect size used is Phi: small effect $\geq 0.10$ but less than 0.30.) 


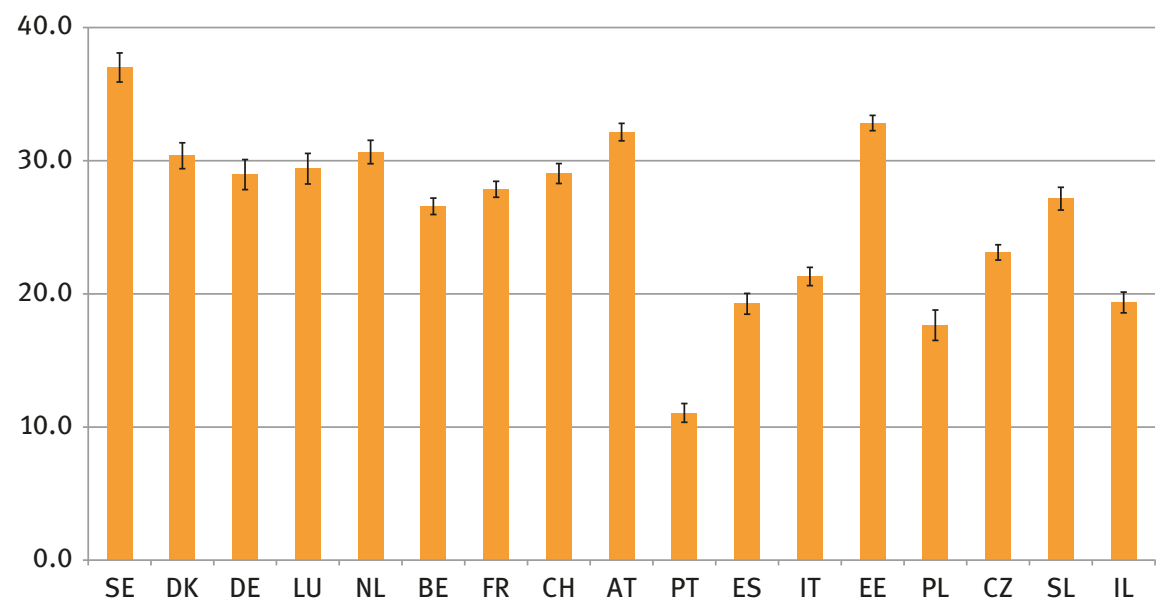

Figure 25.1: Percentage of people aged 50+ and living alone by country (weighted) at baseline. Note: Brackets denote standard errors, $N=57,955$ (unweighted). Wave 4 data used for countries AT, DE, SE, NL, ES, IT, FR, DK, CH, BE, CZ, PL, PT, SL and EE; Wave 5 data for countries IL and LU.

Source: SHARE Wave 4 and 5 release 6.1.0.

The results of the longitudinal analysis are shown in Table 25.1. As may be seen, when loneliness was not taken into account, living alone in five countries was associated with poor physical health (Austria ( $\beta-0.04, \mathrm{p}=0.007)$, Germany $(\beta-0.04, p=0.034)$, Sweden $(\beta 0.05, p=0.006)$, Denmark $(\beta 0.05, p=0.009)$ and Belgium $(\beta-0.03, p=0.014))$ and in three other countries with better physical health (Spain $(\beta$ 0.03, $p=0.061)$, Slovenia $(\beta 0.04, p=0.034)$ and Estonia $(\beta 0.04, p=0.001)$ ). When loneliness was included in the longitudinal regression, living alone had a positive effect on physical health in five countries (Spain ( $\beta 0.06, p>0.001)$, Italy $(\beta 0.05, p=0.003)$, France $(\beta 0.03, p=0.033)$, Slovenia $(\beta 0.05, p=0.009)$ and Estonia $(\beta 0.05, p>0.001))$. Thus, there are important differences when loneliness is introduced. In Austria, Germany, Sweden, Denmark and Belgium, living alone initially had a negative effect on physical health. However, when loneliness was considered, this effect was no longer significant. In Spain and Estonia, the effect continued to be significant. In addition, the effect of living alone in France and Italy became positively significant when loneliness was introduced and, in Slovenia, the positive effect remained.

Regarding mental health, living alone was significantly associated with having depression in 15 countries when loneliness was not yet taken into account (Model 1). After taking loneliness into account in Model 2, a significant negative association emerged in five countries (Austria, Sweden, Spain, Italy and France); that is, living alone, net of loneliness, was related to less risk of depression. 
Table 25.1: Effects of living alone on physical and mental health: Longitudinal linear mixed models by country.

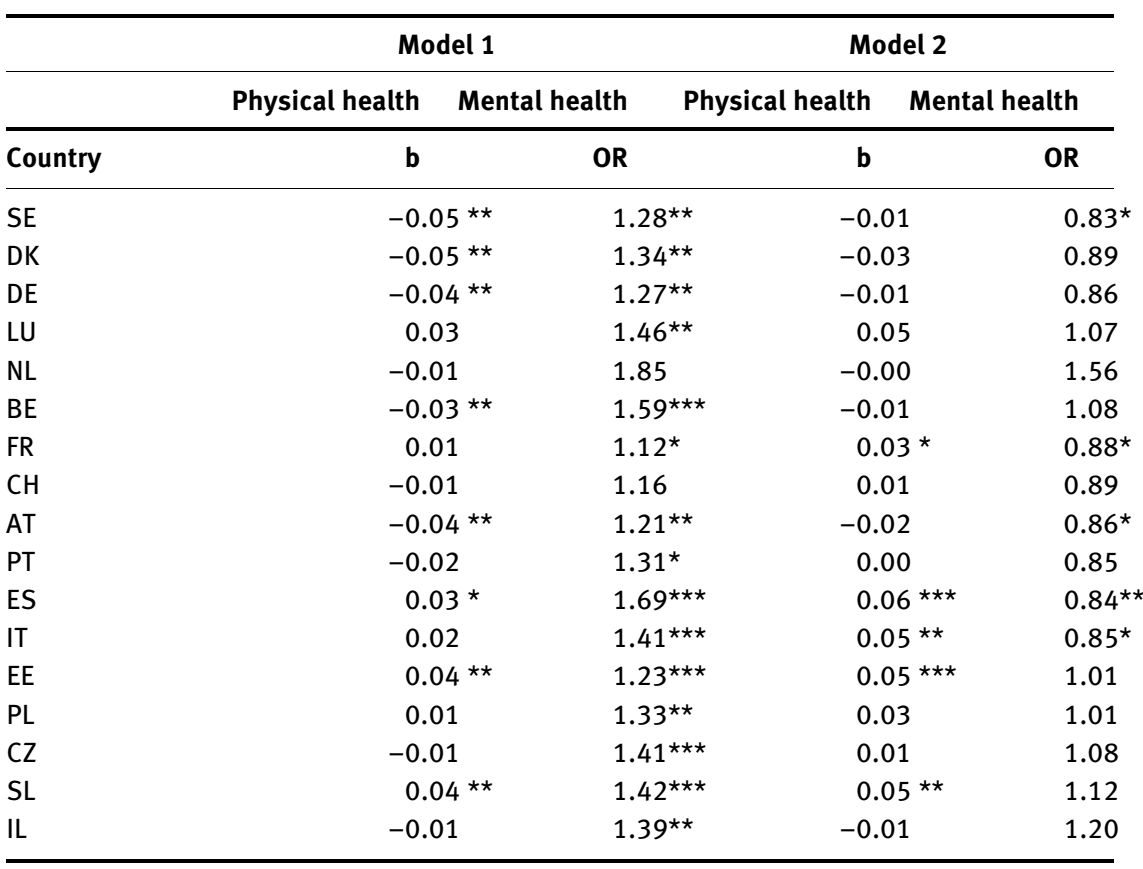

Significance: ${ }^{\star \star *}=1 \% ;{ }^{* *}=5 \%$; ${ }^{\star}=10 \%$.

Note: Mixed models; Model 1: 175,717 observations for 99,021 individuals; Model 2: 163,412 observations for 98,632 individuals. Model 1: adjusted for age, gender, retirement status, financial distress, ISCED, health, number of visits to the doctor, physical inactivity, number of activities done and living alone; Model 2: adjusted to variables of Model 1 plus loneliness.

Source: SHARE Wave 4, 5, 6 release 6.1.0.

In the remaining 10 countries that presented risk of depression when loneliness was not considered, living alone became non-significant with the introduction of this variable. Consequently, when loneliness is adjusted in the model, living alone does not have a negative effect on mental health, and in some countries, it even has a protective role.

\subsection{Conclusions}

Our results show a wide variation in the prevalence of solo living among older Europeans. Solo living is more prevalent in northern, western and eastern 
countries, in contrast to southern countries, Poland and Israel. Moreover, the longitudinal regressions revealed that when loneliness is considered, living alone is not necessarily detrimental to health, specifically mental health. Thus, the findings underscore the important role of loneliness in the association between solo living and health.

In conclusion, these findings have implications for public policies in Europe and Israel. They make it clear that living alone at an older age is a public health concern. However, they also suggest that the negative effect of living alone is driven primarily by loneliness. Therefore, attention should be paid to older people living alone and, especially, to the feelings of loneliness that might be experienced by the older population. European countries should develop and implement public programmes and policies that are geared to reducing the sense of loneliness in ageing cohorts. Reducing loneliness can promote better physical and mental health among older people, improve their quality of life and help reduce burgeoning healthcare costs.

\section{References}

Courtin, E. and Knapp, M. (2017). Social isolation, loneliness and health in old age: a scoping review. Health \& Social Care in the Community, 25(3),pp.799-812.

Park, N., Jang, Y., Lee, B. and Chiriboga, D. (2017). The relation between living alone and depressive symptoms in older Korean Americans: do feelings of loneliness mediate? Aging \& Mental Health, 21(3),pp.304-312.

Ploubidis, G. and Grundy, E. (2011). Health Measurement in Population Surveys: Combining Information from Self-reported and Observer-Measured Health Indicators. Demography, 48(2),pp.699-724.

Shiovitz-Ezra, S. (2015). Loneliness in Europe: do perceived neighbourhood characteristics matter? In: A. Börsch-Supan, T. Kneip, H. Litwin, M. Myck and G. Weber, ed., Ageing in Europe - Supporting Policies for an Inclusive Society. Berlin: de Gruyter.

Victor, C., Scambler, S., Bond, J. and Bowling, A. (2000). Being alone in later life: loneliness, social isolation and living alone. Reviews in Clinical Gerontology, 10(4),pp.407-417. 